\title{
A Novel Video Compression Technique Using Discrete Transforms
}

\author{
Pratik Ratanpara, Asst. Prof. Sagar Virani \\ Department of Computer Engineering, V.V.P. Engineering College, Rajkot, Gujarat, India
}

\begin{abstract}
Nowadays multimedia using video has entered in many applications such as medical, electronic industrial, Electronic Patient Recording, business applications, video conferencing, smart phones, video telephony, digital cameras, and tablets. In multimedia there is a practical need to store, retrieve and transmit for vast amount of digital data; this need can be accomplished by using the terminology called compression. This system is trying to provide a better balance between quality and compression ratios by the visual perception of the decoded video quality remains satisfactory. A video is comprised of series of frames; there will be redundancy between the adjacent frames known as temporal redundancy, to achieve the video compression this redundancy can be exploited by using motion estimation and compensation technique. MPEG-4 has some limitation for compression ratio whereas proposed system has more compression ratio than MPEG-4.
\end{abstract}

Keywords: Arithmetic Coding, DCT, Discrete Wavelet Transform, motion vectors, PSNR.

\section{Introduction}

The video application has become very increasingly popular in medical, [6] mobile applications such as cellular phones and personal computer or laptop. However, due to inherent data intensity of video, transmitting and storing raw video data become difficult. With the limited storage and limited bandwidth capacity, it must be compressed to a transportable size and finally decompressed to reach the destination. For compression purpose, there are two well-known video compression and video decompression algorithms those are Run Length Encoding (RLE) and Arithmetic Coding (AC).[1] The advantage of these compression techniques is that it works for all types of video files for compress and decompress. Video is an infatuation that uses the properties of eye.[6] A video is comprised from series of frames. Eye senses frame from video for $1 / 30$ th of a second. Therefore, video compression algorithms use many techniques and concepts of image compression algorithms, like JPEG (joint photographic expert group).[5]

Compression has two types named lossless and lossy.[1] Unnecessary information is removed to reduce bit in lossy compression. But in lossless compression statistical redundancy is eliminating to reduce bits. Information is not lost in lossless compression.[6] In lossless compression result, original data can be reconstructed exactly same as it was before compression. In digital world, a lossless compression data after decompression it should match original data bit by bit. The compression of lossy, boasts high compression for visual data, but reconstructed data is not same as the original data. It produces high quality digital video, but it requires a lot of data.[1]

Motion estimation technique, Adaptive Rood Pattern, is used for detecting match motion block into next frame to reduce redundancy. Section II focused on video encoder, section III focused on video decoder, section IV gives parameters for measurement, section V gives some experimental results and section VI focused on conclusion.

\section{Video Encoder}

The video compression system contains video encoder at transmitting side. Video encoder encode input video to bits need to transmit. The working of encoder is figured by fig. 1 and explained step by step below.

\section{Step 1:}

Video is collection or group of images. So that first step is to convert video into sequence of frames that are not other than images sequence. In second step 10 frames that are in sequence are selected for further operation that is called group of pictures.

Step 2:

This next step will be to resample or down sample selected frames using bicubic interpolation. So that, the size of frames are decrease and time required for further processed will be saved.

Step 3:

Then at fourth step, group of picture is converted into some sequence like IBBPBBPBBI using compensation technique and motion estimation technique. 
Step 4:

Compute Compased image from Vector to Pridect next frame and error is calculated that will add in decoding process of that frame to currect error.

Step 5:

The further step is to process motion compensated frames using DWT, discrete wavelet transform, after this operation we get four components that are: Approximate, Vertical, Horizontal and Diagonal.

Step 6:

From these four components only one component approximate component is transmitted. So DCT, discrete wavelet transform, of it is taken.

Step 7:

We get coefficients of DCT of image. So we need to further process these components using standard quantization matrix.

\section{Step 8:}

In next step quantized coefficients that are scanned in zig zag manner.

Step 9:

The process of encoding for these coefficients is treated using Arithmetic coding for the better compression.[4] Bit stream of the coder is transmitted or stored on any medium.

\section{Step 10:}

At last step is to form and transmit the bit-stream.

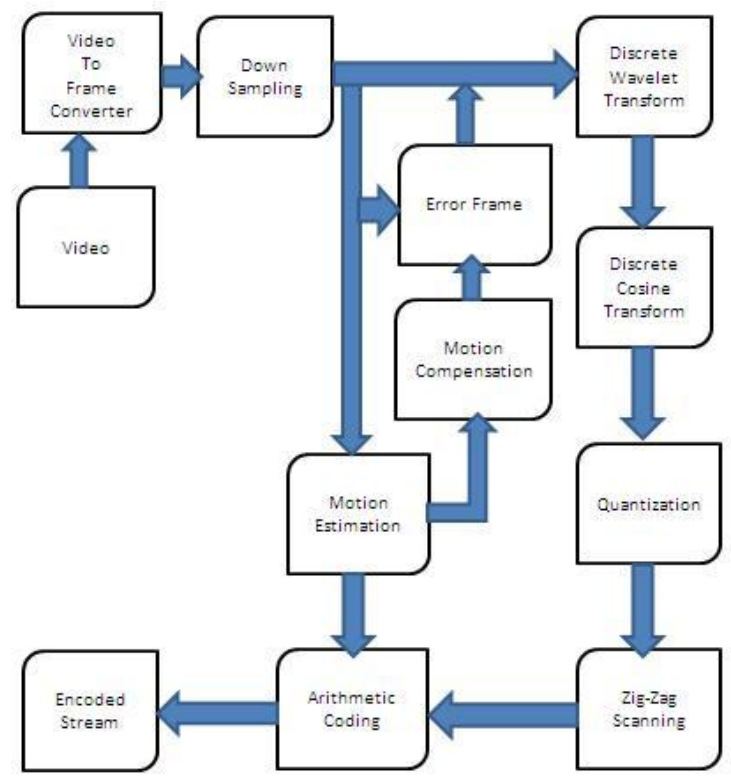

Fig. 1 Video Encoder

\subsection{Down Sampling}

In first step video is converted into frames. Frames are noting but images. All frames are down sampled using bicubic interpolation method. So, the size of video frame is decrease about $75 \%$. It is also useful in calculating further process for saving time. Then after, they are grouped into IBBPBBPBBI frames.

\subsection{Temporal Compression}

In video sequence frames are mostly contain same information with each other. So, frames can be predicted one from other which gives us temporal compression into the video. So, from this functionality there are three kinds of frames can be generated from group of picture: Iframe, Pframe and Bframe. [5] 


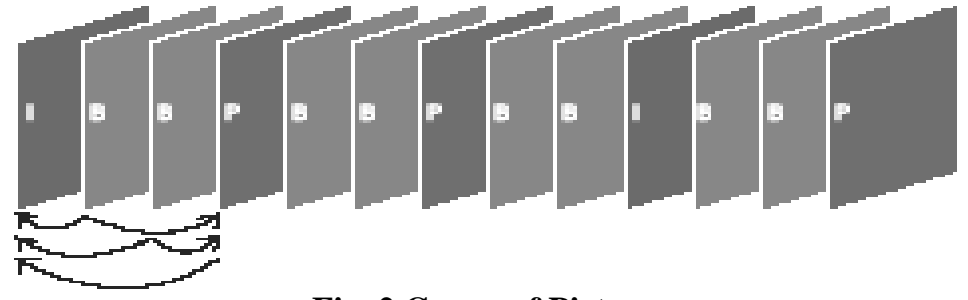

Fig. 2 Group of Pictures

Some blocks may be predicted from blocks of previous frames. Frames which contain such predicted blocks are known as P-frames. There might be error in decoder if the first frame of a video is lost. There might be error in decoder if sequence of $\mathrm{p}$-frame gets error. As a solution of this situation a complete frame which does not depend on any other frame need to transmit periodically. These original frames are called I-frames or intracoded. Other than this two frames that borrows blocks from both side before and after to it. These frames are known as B-frames.

Further improvement in saving few more bits is to encode motion vectors. Thus only portions of a frame that moves or blocks of a frame that can be borrowed from other blocks in previous frames in video, are coded with fewer bits. For this purpose, $16 \times 16$ macro blocks are divided into the $8 \times 8$ pixel blocks.[2][5]

Some macro blocks are same as previous macro block means they do not change. These are not recoded in subsequent frames to saving size. Using P-frames, the encoder searches previous frame that are close match to current macro block for location of other macro block. If no matching blocks are found in neighboring block, the current macro block is fully coded and also DCT coefficients are fully coded.[2]

If matching block is found in search portion, motion vector is used to point for the similar blocks instead of full coefficients. When decoding current frame, the decoder uses information using motion vector from the other frame.

\subsection{Adaptive Rood Pattern Search}

Adaptive Rood Pattern Search algorithm uses the fact that frames that contain general motion is usually compatible, that means if some of macro blocks surrounding current macro block are moving in some particular direction so then there might be a high probability for the current macro block might have similar motion vector same as surround vector. ARPS uses the motion vector of its immediate left macro block for own motion vector to predict.[3]

ARPS also tests at rood pattern for distributed points to checking location that pointed by predicted motion vector, where their distance are called a step size that is maximum out of $\mathrm{X}$ and $\mathrm{Y}$. $\mathrm{X}$ is the $\mathrm{x}$ coordinate and $\mathrm{Y}$ is $\mathrm{y}$ coordinate of that predicted motion vector. This process of searching rood pattern is always the staring step. That puts directly the searching process in the area where there may be high probability for finding good matching block. Then, the point which has least weight it becomes the origin of subsequent search steps. So the search pattern is converted in to SDSP. This procedure continues with SDSP until center of SDSP is found as least weighted point.[3]

A small improvement into this algorithm could be for checking for Zero motion Prejudgment, if least weighted points are already at the center for that rood pattern, by which search is stopped at half way. [3] The big advantage of this changes over DS if predicted motion vector is at $(0,0)$, then computational time does not waste in doing LDSP. For that continue directly with SDSP.[3]

Other than this, if predicted motion vector is at far distance from the center, for this time again ARPS save computations time by using SDSP and directly jumping, but DS takes time for calculating LDSP. Care must be taken for not repeat computations were checked earlier at points. Algorithm must have to avoid this double computation at that point. Step size is fixed 2 pixels for blocks in first column of the frame.

\section{Video Decoder}

The video compression system contains video decoder at receiver side. Video decoder decodes input data bits and produce video data. The working of decoder is figured by fig. 3 and explained step by step below.

\section{Step 1:}

In the first reverse step bit stream of MPEG is decoded with Arithmetic decoder and converts it to original coefficients DCT.

Step 2:

These coefficients for DCT are quantized using quantization matrix to get original DCT coefficients. 
Step 3:

At this stage inverse DCT is taken that give approximate component of frame.

\section{Step 4:}

Zeros are appended to horizontal, diagonal and vertical component of frame to perform inverse DWT. Take inverse DWT of four components.

Step 5:

Then the motion estimation algorithm is used to get motion compensated frame and error frame is combined to get original frame.

\section{Step 6:}

Then component is up sample or re-sampled to original form.

\section{Step 7:}

Finally the sequence of frames is converted to video which is final compressed video.

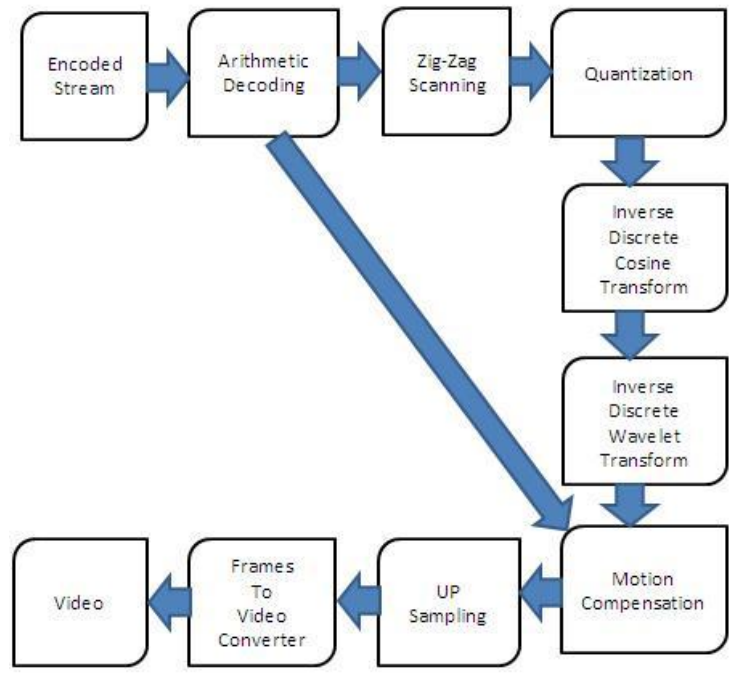

Fig. 3 Video Decoder

\section{Measuring Parameters}

We use manually following parameters for the sake of subjective measure of degradation of video after the compression.

$$
\begin{gathered}
\text { PSNR }=10 * \log \frac{255^{2}}{N S E} \\
\operatorname{MSE}=\frac{1}{N^{2}} \sum_{i=0}^{N-1} \sum_{j=0}^{W-1}\left(C_{i j}-R_{i j}\right)^{2}
\end{gathered}
$$

Where $\mathrm{N}$ is size of side of macro bock, $\mathrm{R}_{\mathrm{ij}}$ and $\mathrm{C}_{\mathrm{ij}}$ are pixels that are going to compare in reference macro block and current macro block. The signal to noise ratio called PSNR given by equation (1) that is term used for calculating power between corrupting noise and maximum power of signal and return ratio between them. PSNR is measured terms of decibel scale because many signals have dynamic range.

Also find the compression ratio between two videos, the original one and the compressed one coming out of video encoder, which is measured by equation (3) as below: [2]

$$
\mathrm{CR}=100 * \frac{\text { Compressed data rate }}{\text { Uncompressed data rate }}
$$

\section{Experimental Results}

There are various frames of original video before encoding are shown in Fig. 4 and decoded frames at receiver side are shown in Fig. 5. 


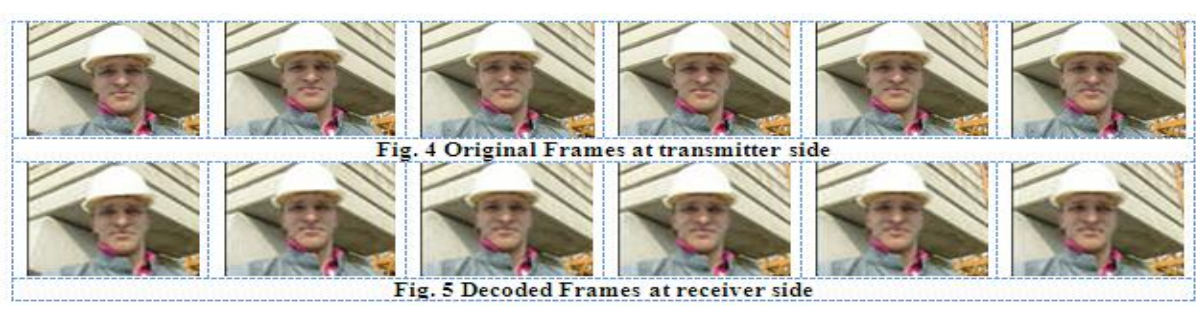

This proposed algorithm is implemented on various data sets and collect results as listed in Table. 1. We also get PSNR values for this data set for first 10 frames as figured in fig. 6.

Table 1. Compression Ratio for different videos

\begin{tabular}{|c|c|c|c|c|c|c|}
\hline Video Data & $\begin{array}{c}\text { Size } \\
(\mathrm{Mb})\end{array}$ & Frames & $\begin{array}{c}\text { Compression } \\
\text { Ratio }(\%)\end{array}$ & $\begin{array}{c}\text { Compressed } \\
\text { File }(\mathrm{Kb})\end{array}$ & $\begin{array}{c}\text { Time } \\
(\mathrm{sec})\end{array}$ & PSNR \\
\hline Foreman.avi & 4.35 & 60 & 94.3 & 250 & 17.47 & 24.19 \\
\hline Overhead.avi & 6.96 & 96 & 95.9 & 295 & 33.51 & 23.04 \\
\hline Viplane.avi & 5.22 & 72 & 94.5 & 294 & 22.18 & 24.78 \\
\hline
\end{tabular}

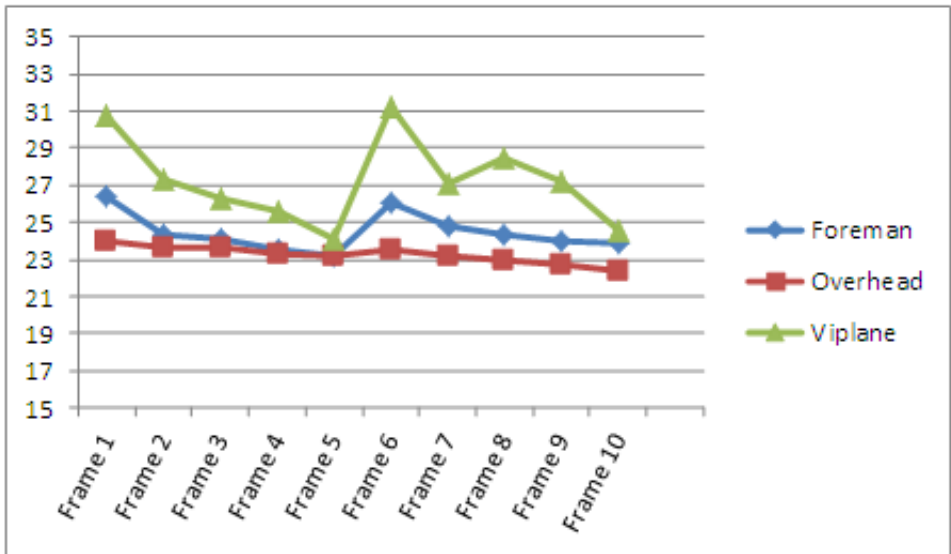

Fig. 6 Graph of PSNR v/s Frames

\section{Conclusion}

This system is trying to provide a better balance between quality and compression ratios by the visual perception of the decoded video quality remains satisfactory. After applying proposed algorithm we can achieve about $94 \%$ compression ratio and also average PSNR value is around $24 \mathrm{~dB}$ for various tested video data. This PSNR value gives enough visibility that human can perceive the video easily. This technique is better in terms of compression ratio than MPEG that use only DCT but it required little more time. Compressed video data can successfully decrease about $94 \%$ bandwidth required to transmit.

\section{Acknowledgements}

There are many people who inspired and helped in making research successful. We thank to staff member of VVP Engineering College, Rajkot, to give valuable guidance to us about research and publication. I am also thankful to Sagar Virani, Assistant Professor, VVP Engineering College, Rajkot, to encourage, advice and support. Last, but not least our special thanks to our institute, VVP Engineering College, Rajkot, to giving this opportunity in great environment.

\section{References}

[1] Mozammil, S. M. Zakariya and M. Inamullah, Analysis of Video Compression Algorithms on Different Video Files, IEEE transaction on fourth International Conference on Computational Intelligence and Communication Networks, 2012.

[2] Suchitra Shrestha and Khan Wahid, Hybrid DWT-DCT Algorithm for Biomedical image and Video compression Applications, IEEE transaction on tenth International Conference on Signal processing, 2010.

[3] Vanshree Verma, and Sr. Asst. Prof. Ravi Mishra, A novel Adaptive Rood Pattern search Algorithm, IOSR Journal of Electrical and Electronics Engineering, Volume 4(4), 2013, pp. 14-18.

[4] Jafari, A. and Rezvan, M. and Shahbahrami, A Comparison between Arithmetic and Huffman Coding Algorithms, IEEE sixth Iranian Machine Vision and Image Processing Conference, October 2010, pp. 248-254.

[5] Jaemoon Kim, Jungsoo Kim and Chong-Min Kyung, A Lossless embedded compression algorithm for High Definition video coding, ninth International Conference on Fuzzy Systems and Knowledge Discovery, 2009

[6] Nedia NOURI, Denis ABRAHAM, Jean-Marie MOUREAUX, Michel DUFAUT,Jacques HUBERT and Manuela PEREZ, Subjective MPEG2 Compressed Video Quality Assessment: Application to Tele-Surgery, IEEE fifteenth International Conference on Computational Science and Engineering, 2010. 